\title{
TWO-DIMENSIONAL DEFORMATION PATTERNS IN SEA ICE
}

\author{
By BJÖRN ERLINGSSON
}

(Geophysical Institute, University of Oslo, Blindern, N-0315 Oslo 3, Norway,

and Norsk Polarinstitutt, N-1330 Oslo Lufthavn, Norway)

\begin{abstract}
The mechanical behaviour for the geometrical properties of two-dimensional deformation has been investigated. The Mohr formulation of failure, which is based on the concept of slip at the plane of weakness, is used in this study. It is shown that alteration of the shear stress $\tau_{\mathrm{n}}$ at the plane of weakness requires self-similarity of features in deformation patterns. A general relationship between the angle of internal friction and the breaking angle of deformation patterns is derived. Using imagery data, this relationship is used to find the angle of internal friction from selected sea-ice deformation patterns. The value obtained for this angle is $\Phi=15^{\circ} \pm 2^{\circ}$ for deformation on a scale from $100 \mathrm{~m}$ to $100 \mathrm{~km}$.
\end{abstract}

\section{INTRODUCTION}

Fracture patterns appear as the most prominent feature of materials that have been deformed permanently. Features such as slip lines, crevasses, and faults are found on a wide range of geophysical scales resulting from deformation in sea ice, glaciers, and in the Earth's crust. These patterns often exhibit a uniform and systematic character which signifies the state of the rheological environment in the material during a particular deformation. The main question addressed here is: what properties of the material can one deduce from observations of a deformation pattern?

The formulation of constitutive equations which accurately describe the mechanical behaviour of materials undergoing deformation is an outstanding problem in geophysical continuum mechanics. Present experimental efforts are difficult to interpret and of ten give inconclusive results. In general, the rigorous interpretation of geophysical phenomena requires the knowledge of mechanical properties and fragmentation processes of the substance being investigated. A model for the drift of geophysical materials (e.g. sea ice, glacier ice, or rock in the Earth's crust) must necessarily account for the behaviour which is responsible for internal forces. This study is concerned with sea-ice deformation and in general its relation to sea-ice morphology, fragmentation, and fine-scale motions.

A reasonable way to make progress is to investigate the implications of various sets of equations which have been proposed for describing the stress in a material. As a result, one may hope to derive a theoretical description which can be tested against the behaviour of real deformable materials in real situations. This can be accomplished by using the illustrative Mohr formulation of the stress state in a deformed material where the extension of the results from two dimensions to three dimensions is trivial. The results lead to geometrical self-similarity of features in the deformation patterns which are scale-invariant. This is especially useful, since no $a$ priori assumptions regarding the material properties of the media need be made. The concept of self-similarity provides a useful tool for the geometrical description of deformation patterns, which simplifies the interpretation of measurements.

\section{BASIC CONCEPTS}

The analysis of stress is essentially a branch of statics, since one attempts to resolve the forces at any point in a material into useful quantities. The Mohr formulation of stress provides a simple and physically meaningful tool which can be applied to two- or three-dimensional stresses with no loss in generality. Sea-ice deformation can be considered as two-dimensional in either a horizontal or vertical plane. Such deformation is caused by external forcing that results in relative motion (in the horizontal plane) and ridging and rafting (in the vertical plane). A brief review of the basic concepts of stress analysis is necessary in order to understand fully its application to problems involving sea-ice deformation (see for example Jaeger, 1969).

The occurrence of deformation can be described in terms of observable strains as a permanent disturbance of a regular structure, whereas stresses are not directly observable. Therefore, a deformation criterion involving strain rather than stress would be more useful. Despite many attempts, no satisfactory strain theory has been developed. The critical strains are more or less dependent on the length scale over which a particular disturbance is measured. The Mohr theory assumes that failure (i.e. fracture and/or flow) occurs when the shear stress attains a critical value which is related to the normal stress. We describe this critical value by a functional relationship which is independent of the direction of failure. Typically, one defines the strain in terms of the slip across the plane of weakness. Introducing the state variables $A_{i}$ (e.g. density, temperature, etc.), this relation between shear and normal stress is expressed as

$$
\left|\tau_{\mathrm{n}}\right|=f\left(\sigma_{\mathrm{n}}, A_{i}\right)
$$

where $\tau_{\mathrm{n}}$ is the shear stress and $\sigma_{\mathrm{n}}$ is the normal stress. The expression $f\left(\sigma_{\mathrm{n}}, A_{j}\right)$ is called the Mohr-envelope or the yield curve, and is a generalization of the maximum shear-stress theory which dates back to Coulomb in 1773 (Jaeger, 1969). The linear version of Equation (1). (the so-called Coulomb-Navier criterion) completely describes the physics of constant friction between sliding surfaces, and has been successfully used in many of the problems of geological faulting. The interpretation of Equation (1) is crucial in the verification of the mechanical properties of the materials under study. In a two-dimensional stress state, the shear stress and the normal stress are related to the isotropic pressure, $\Pi$, and the shear, $\Gamma$, through the equation

$$
\tau_{\mathrm{n}}^{2}+\left(\sigma_{\mathrm{n}}+\Pi\right)^{2}=\Gamma^{2} .
$$

Equation (2) describes the Mohr circle in the $\left(\sigma_{n}, \tau_{n}\right)$-plane (Fig. 1). It is well known that the shear and the normal stress, $\tau_{\mathrm{n}}$ and $\sigma_{\mathrm{n}}$ on a line $\mathrm{L}$, whose normal $\mathbf{n}$ makes the angle $\psi_{0}$ with the principal axis $\sigma_{1}$, is related to the shear and isotropic pressure, $\Gamma$ and $\Pi$, such that 

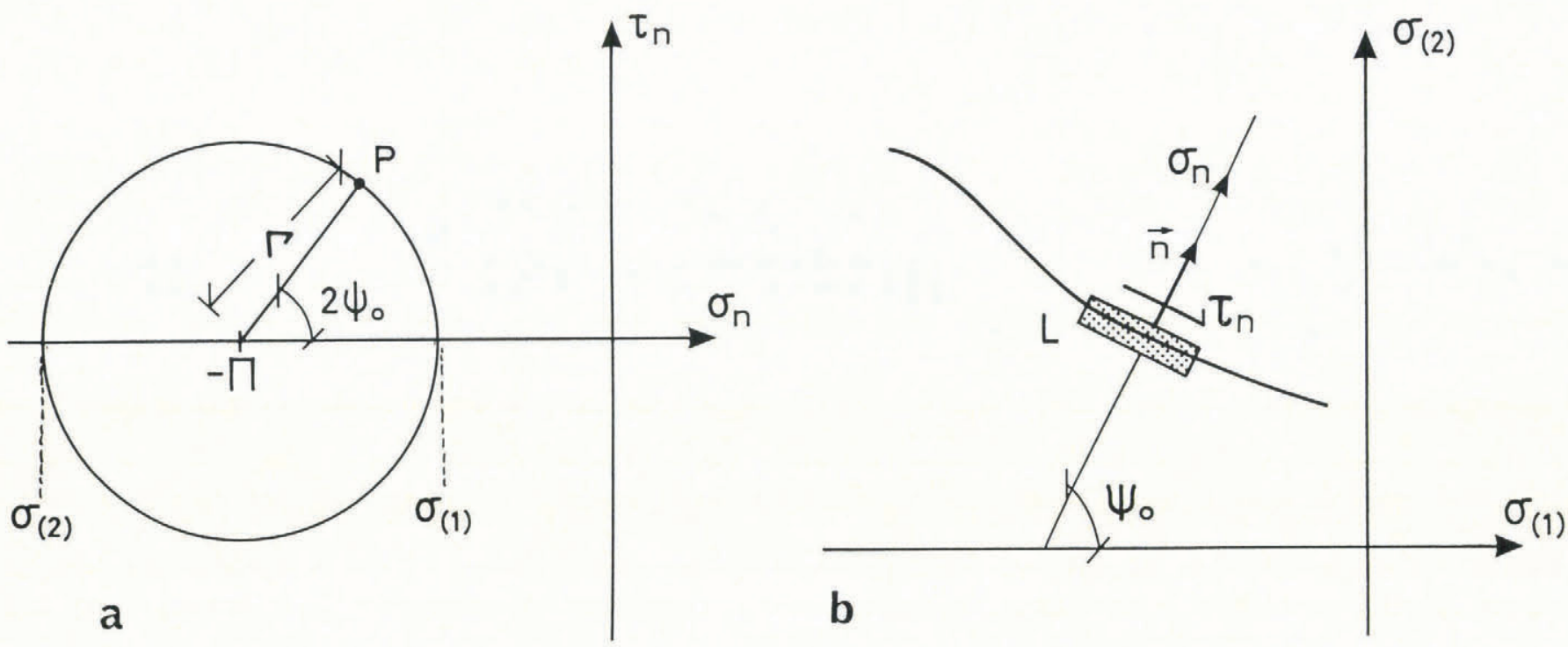

Fig. 1. a. The Mohr circle in the $\left(\sigma_{n}, \tau_{n}\right)$-plane.

$b$. The configuration of the shear and normal stress on the line $L$ in the principal stress plane.

$$
\begin{gathered}
\sigma_{\mathrm{n}}=-\Pi+\Gamma \cos \left(2 \boldsymbol{\psi}_{0}\right), \\
\tau_{\mathrm{n}}=\Gamma \sin \left(2 \boldsymbol{\psi}_{0}\right) .
\end{gathered}
$$

The shear and isotropic pressure are related to the principal stresses (greatest and least normal stresses) $\sigma_{1}$ and $\sigma_{2}$ through the following equations

$$
\begin{gathered}
\Pi=-\left(\sigma_{1}+\sigma_{2}\right) / 2, \\
\Gamma=\left(\sigma_{1}-\sigma_{2}\right) / 2 .
\end{gathered}
$$

The principal directions correspond to the directions of the greatest and least normal stresses. They are always separated by an angle of $\pi / 2$. In order to avoid unnecessary confusion, this set of principal directions will be considered as one principal direction co-linear with the $\sigma_{1}$-axis. When the shear strength $\Gamma$ (maximum shear stress) is represented as a function of the isotropic pressure $\Pi$ and state variables $A_{i}$, one can express the yield condition in a different functional form. It is related to the yield criterion for the shear stress (as a function of normal stress) through Equations (2) and (3). Both representations of the yield criterion describe the same state of stress in a substance undergoing deformation. A useful mechanical property related to both yield conditions is the internal friction, $\tan (\Phi)$, which is defined as the rate of change in positive shear stress with compressive normal stress when state variables are held constant (Kingston and Spencer, 1970).

$$
\tan (\Phi) \equiv-\left.\frac{\mathrm{d} f}{\mathrm{~d} \sigma_{\mathrm{n}}}\right|_{i} .
$$

Here, $\Phi$, the so-called angle of internal friction, may be expressed in terms of the shear strength and the isotropic pressure. Using Equations (3) and (5), one obtains

$$
\left.\frac{\mathrm{dr}\left(\Pi, A_{i}\right)}{\mathrm{d} \Pi}\right|_{A_{i}}=\sin (\Phi) \text {. }
$$

Equation (6) predicts that the shear in a substance $\left(\Gamma\left(\Pi, A_{i}\right)\right)$ cannot increase or decrease more rapidly than the isotropic pressure since $|\sin (\Phi)|<1$. Yield conditions violating this inequality have little physical meaning for an actual stress state of a substance. Some yield conditions assumed for sea ice unfortunately do not take this into consideration. Since the geometrical properties of the deformation pattern will be related to the angle of internal friction, one can gain information concerning the criterion for deformation using Equations (5) or (6).

\section{THE SELF-SIMILARITY OF DEFORMATION PATTERNS}

The notion of self-similarity has been evoked in an attempt to gain insight into various geophysical problems. The fractal or self-similar properties of deformation patterns such as fragmented sea ice (Rothrock and Thorndike, 1984; Matsushita, 1985) and tectonically evolved land forms (Mandelbrot, 1975) have been investigated. In describing the geometrical properties of sea-ice keel profiles, Rothrock and Thorndike (1980) showed that the thickness distribution exhibits fractal or self-similar properties. The idea of self-similarity is also a useful concept in the description of the geometrical properties of sea ice. In this section, the self-similarity of deformation patterns will be related to the mechanism of failure.

The role of self-similarity in the study of deformation patterns concerns the angular relation of the various features observed. It is found that a single angle (or a multiple thereof) relates certain features in the deformation pattern to each other. This angle is related to the yield stress and strain at a particular point in the material. The angular relationship is deduced from the Mohr theory of failure and is length-scale invariant. According to this theory, the tangential components of stress are anti-parallel about the plane of weakness which constrains the orientation of the principal stress (on each side of the plane), thereby fulfilling the yield criterion. In two dimensions, a slip line going through one point is the line in the physical plane (the $x, y$-plane) where the shear and normal stress satisfy the yield criterion. The shear and normal stress corresponding to a particular isotropic pressure $\Pi$ are given at the yield points $P_{1}$ and $P_{2}$, where the Mohr envelope is tangential to the Mohr circle (see Fig. 2). The slip lines $S_{1}$ and $S_{2}$ in the principal plane correspond to these yield points and the angles $\psi_{1}$ and $\psi_{2}$ define the direction of the slip-line normals (e.g. Kingston and Spencer, 1970). The directions of slip lines $S_{1}$ and $S_{2}$ with respect to the principal direction $\sigma$ are

$$
\theta\left(\sigma, \mathrm{S}_{1,2}\right)=\psi_{1,2}-\pi / 2=-\lambda,+\lambda
$$

where the angle $\lambda$ is related to the angle of internal friction by

$$
\lambda=\pi / 4+\Phi / 2 \text {. }
$$

Suppose one observes a slip line and wishes to deduce the associated principal direction. There are two principal directions which may fulfil the yield condition and generate the observed slip line. One corresponds to the shear stress 

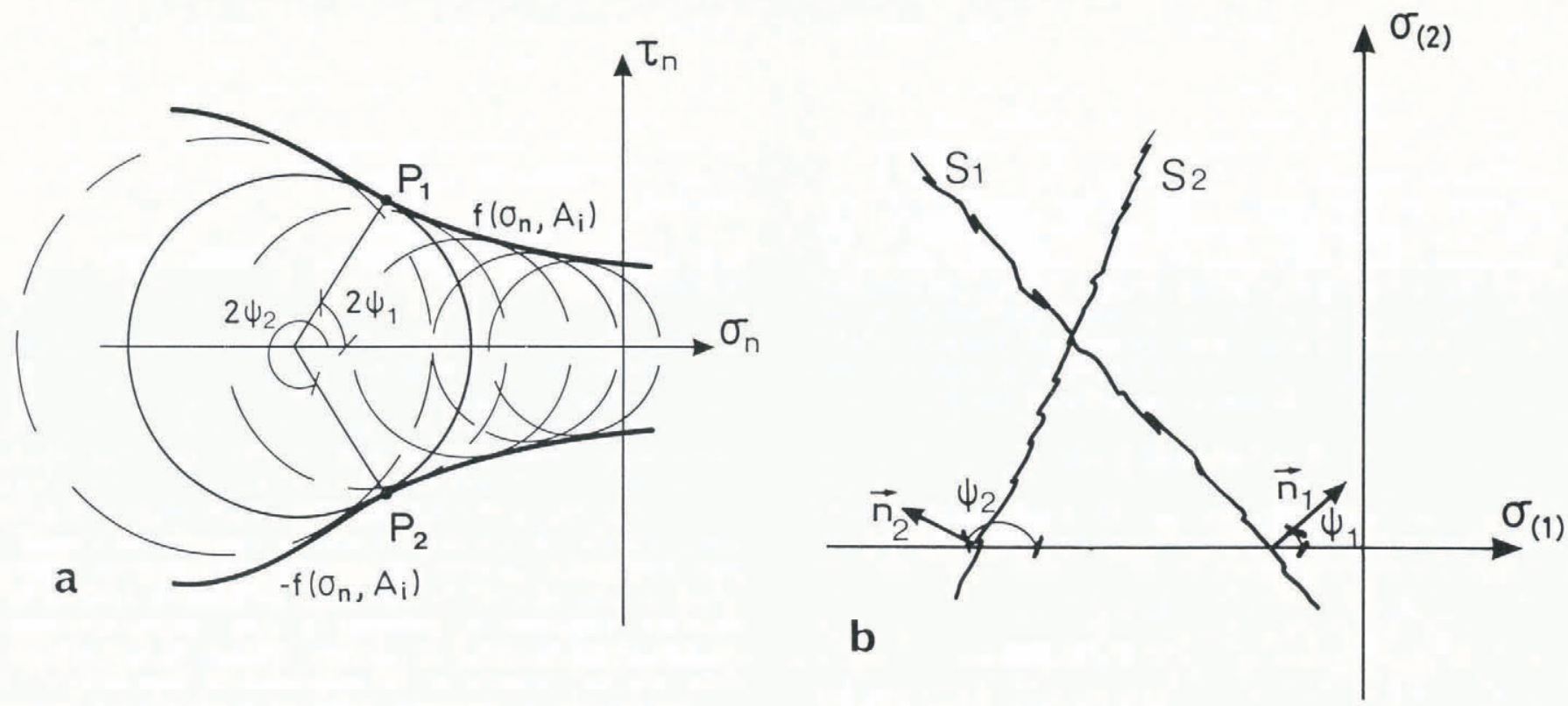

Fig. 2 a. The yield criterion in the $\left(\sigma_{n}, \tau_{n}\right)$-plane.

$b$. The orientation of the slip lines $S_{1}$ and $S_{2}$ in the principal plane.

on the normal side of the slip line, $\sigma^{+}$, and the other corresponds to the reacting shear stress on the other side, $\sigma^{-}$. Their orientations (see Fig. 3) with respect to the slip line $\mathrm{S}$ in the physical plane can be deduced through the use of Equation (7) and are given as

$$
\theta\left(\mathrm{S}, \sigma^{ \pm}\right)=\psi_{ \pm}-\pi / 2= \pm \lambda .
$$

The two principal directions given by Equation (9) correspond to one observed slip line and can generate two sets of slip lines with directions $\theta\left(\sigma^{ \pm}, \mathrm{S}_{1,2}\right)$ dictated by Equation (7). A particular slip-line direction from each set, $\theta\left(\mathrm{S}_{1}, \sigma^{+}\right)$and $\theta\left(\mathrm{S}_{2}, \sigma^{-}\right)$, corresponds to the direction that was originally observed; thus, the remaining directions from each set, $\theta\left(\mathrm{S}_{2}, \sigma^{+}\right)$and $\theta\left(\mathrm{S}_{1}, \sigma^{-}\right)$, respectively make the angles $+2 \lambda$ and $-2 \lambda$ with the slip line originally observed. This can be taken further, and the possible slip-line and principal directions corresponding to a particular state of stress at one point have therefore distinct values. If the angle between an observed feature (e.g. slip line or fracture) and the principal direction it belongs to is known, then the features which belong to the same state of stress can be related to the same principal direction through that deduction. This direction is called the primary direction and is related to the external loads that generate the deformation.

To each possible principal direction $\sigma_{j}$ there is a corresponding slip line $\mathrm{S}_{j}$. Their orientations in the physical plane, $\theta$, are given by

$$
\begin{gathered}
\theta\left(\sigma_{j}\right)=\theta_{0}+2 j \lambda=\theta\left(\mathrm{S}_{j}\right)-\lambda \\
j=0, \pm 1, \pm 2, \ldots
\end{gathered}
$$

where $\theta_{0}$ is the orientation of the primary principal direction $\sigma_{0}$ in the physical plane and $j$ is here called the branching index. The angles between principal directions $\left(\sigma_{j}\right.$ or $\sigma_{k}$ ), slip lines, or any other formations which have a fixed angle with respect to a principal direction $\left(\sigma_{j}\right.$ or $\left.\sigma_{k}\right)$ are given by

$$
\begin{gathered}
\Delta_{i} \theta=2 i \lambda \\
i=0, \pm 1, \pm 2, \ldots
\end{gathered}
$$

where $i$ (here called the breaking index) is the difference in the branching index of the corresponding principal direction (i.e. $i=j-k$ ). This equation gives the angular relation between mechanically similar features in the deformation
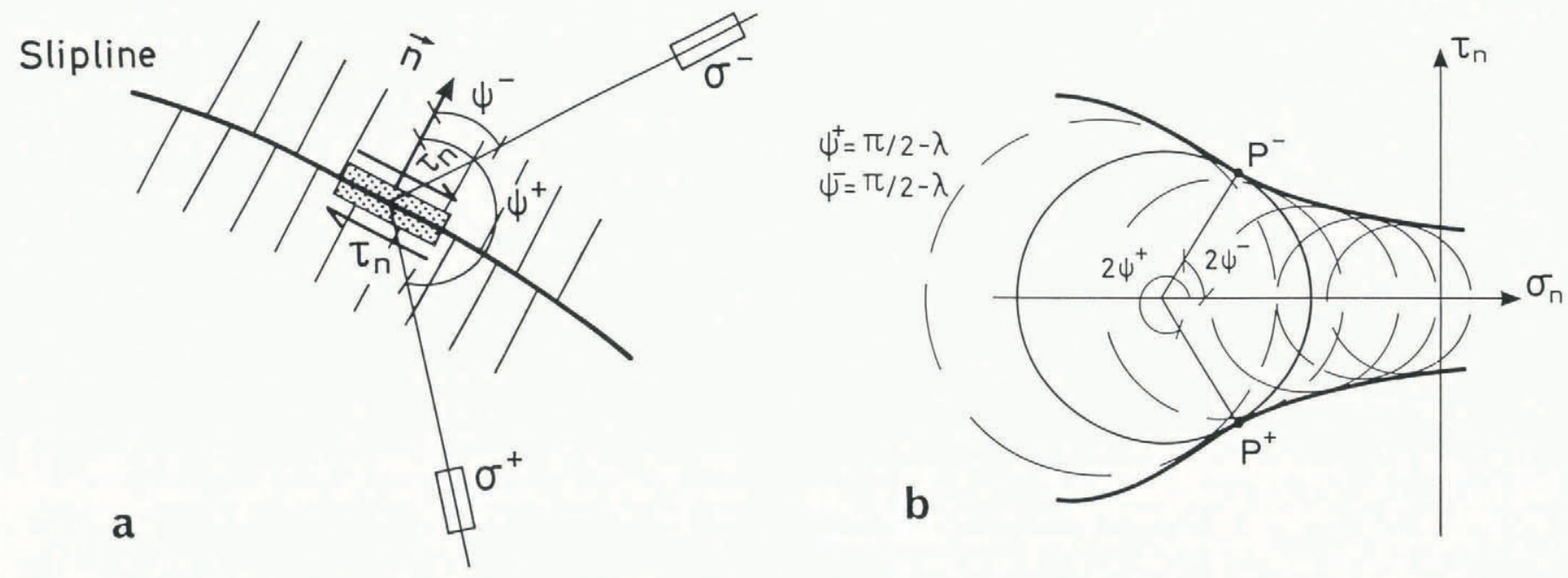

Fig. 3. The configuration of possible principal directions $\sigma^{+}$and $\sigma^{-}$in the physical plane (a) that correspond to the yield points $P^{+}$and $P^{-}$in the $\left(\sigma_{n} \tau_{n}\right)$-plane $(b)$. 
field without any reference to the primary principal direction.

The fractal properties or the self-similarity of the fracture and deformation phenomena, characterized by the Hausdorff or fractal dimension $D$, can be related to measurable mechanical properties of the material being subjected to deformation. The fractal generator of the fracture or deformation pattern, and its placement in the deformation field, must obey the same geometrical rule as is valid for an arbitrary similar feature in the deformation field. Equation (11) gives rise to many possible forms of fractal generator having those geometrical properties. The angle $2 \lambda$ is a simple generator of fractures or other features and has a fractal or Hausdorff dimension $D$ (see Fig. 4)

$$
D=\ln (2) / \ln (2 \sin (\lambda)) \text {. }
$$

In order to examine the angle of internal friction from the viewpoint of the geometrical (or fractal) properties of

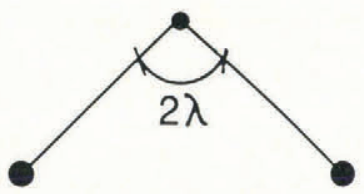

$$
\begin{aligned}
& N=2 \\
& r=1 / 2 \sin \lambda \\
& D=\ln (2) / \ln (2 \sin \lambda)
\end{aligned}
$$

Fig. 4. Fractal generator $2 \lambda$ of fragmentation and deformation.

an observed deformation pattern, one must know the generator and the associated rule of placement. In practice, the generator is generally not directly observable but its associated fractal dimension can be extracted from observable geometrical quantities derived from features in the deformation pattern. On the other hand, measurements of intersection angles between rectilinear features and trends in deformation patterns are easily made. This can be done without any knowledge of the primary principal direction. From Equation (11), the steepest angle between two features having the same relation to principal directions differing in multiple by $i$, here called the breaking index, is given by

$$
\begin{gathered}
\beta_{i}=\min \{|2 i \lambda-1 \pi|\}, \\
i, 1=0, \pm 1, \pm 2, \ldots
\end{gathered}
$$

where $\beta_{i}$ is called the breaking angle. Observations of intersection angles between the rectilinear features of two-dimensional deformation fields provide sets of discrete values for the angle of internal friction as a function of

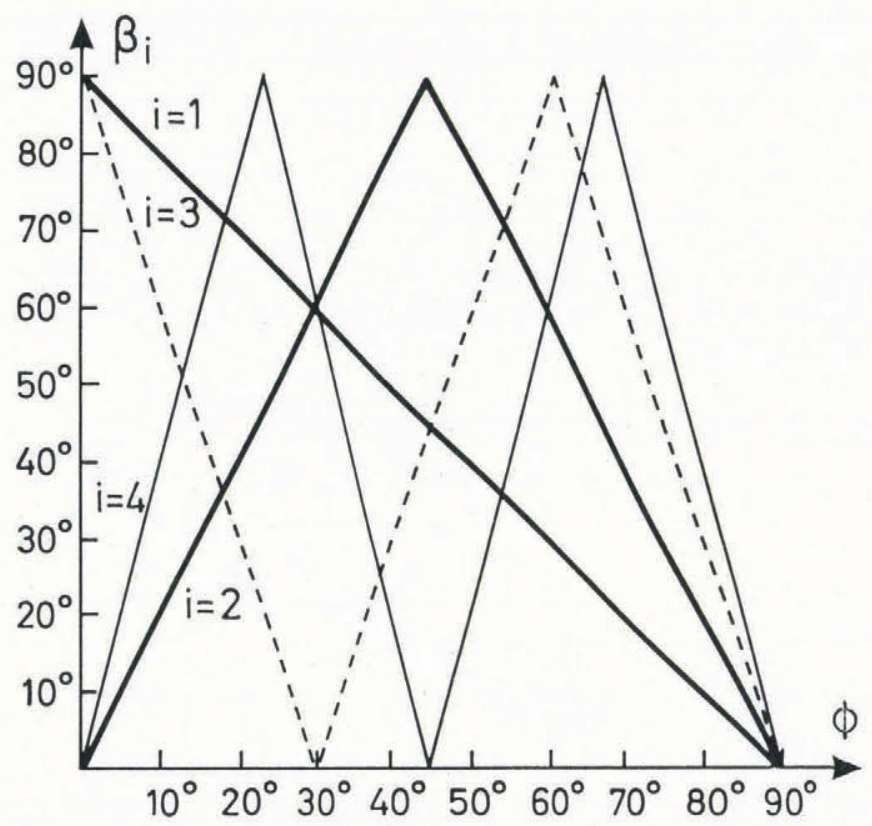

Fig. 5. Breaking angle, $\mathrm{B}_{i}$, as a function of the angle of internal friction, $\Phi$, and breaking indices, $i$. the breaking index $i$ (see Fig. 5). This relationship is useful, since one is primarily interested in finding the mechanical properties of the deformed material from observable geometrical quantities. In a later section, the value for the internal friction, which most accurately fits the observations of sea-ice deformation patterns of different length scales, is examined.

\section{SLIP-LINE AND PRINCIPAL DIRECTIONS AT BOUNDARIES}

The fixed external boundaries of a deformation field are ultimately the most important factors in the verification of its rheological properties. External boundaries of sea-ice deformation fields include interactions between irregular coastlines, the land-fast ice zone, and the near-shore drift ice on the one side, and the offshore ice pack on the other side. These boundaries provide the fundamental frictional control of flow in response to external loads or body forces. The rheological models, which adequately describe their behaviour, are suitable for the entire deformation field. It is therefore important to establish a clear mechanical and rheological interpretation of common features in deformation fields and their relation to different kinds of boundaries.

Boundaries are generally considered as divisions between different physical conditions. For deformation, these conditions are dynamic and kinematic depending on the mechanical conditions and strains. Features in deformation patterns, which are related to the strains or principal directions, represent the kinematic or dynamic conditions in the material. They represent discontinuities, as they are related to different strains or principal directions, and can therefore be considered as internal boundaries. Thus, the concept of a boundary may apply to any feature in the deformation pattern. It is therefore practical to choose a boundary as that feature in the deformation field which has the greatest characteristic length scale. Thus, the choice of a boundary depends not only on the physical situation in the deformation field but also on one's own observational capability. In sea ice, undeformed land-fast ice, large-scale pressure and shear zones, leads and slip lines can be considered as boundaries.

Open fractures, crevasses, or leads in deformation fields imply that the normal stress at the fracture has a value which is a positive maximum $\left(\sigma_{\mathrm{n}}>0\right)$. Thus, the fracture (crevasse or lead) makes an angle $\pi / 2$ with a corresponding principal direction. For a pressure ridge or hummock, this situation is just the opposite. The normal stress along a pressure ridge is at a minimum and is compressive in nature $\left(\sigma_{n}<0\right)$. Thus, a corresponding principal direction is colinear with (along) the pressure ridge. The definition of a shear ridge is less clear in a mechanical sense and a more rigorous definition is needed. A shear ridge may be compared to a strike-slip fault where the principal shear direction is parallel to the slip-line direction.

The orientations of a shear boundary and a pressure boundary are considered as those which have the greatest shear stress and compressive normal stress, respectively (see Fig. 6a and b). The corresponding primary principal direction $\sigma_{0}$ has the intersection angles $\pi / 4$ and 0 with the shear and pressure boundary, respectively. The corresponding intersection angles (using Equation (10)) for the primary slip lines $S_{0}$ are $\Phi / 2$ and $\lambda=\pi / 4+\Phi / 2$. The kinematic slip and the orthogonal non-slip boundary represent a slip line or a strike-slip fault and the normal to it (see Fig. 6c and d). The corresponding primary principal direction (using Equation (10)) $\sigma_{0}$ has the intersection angles $\pi / 4+\Phi / 2$ and $\pi / 4-\Phi / 2$. The intersection angles of tension fractures (openings, leads, or crevasses) are $\pi / 2-\Phi / 2$ and $\pi / 2+\Phi / 2$, respectively. Intersection angles of $\pi / 4$ between open fractures (or crevasses) and the sides of ice flows in glacier outlets are frequently observed (see for example Nye, 1952), implying that the sides act as shear boundaries. The land-fast ice off the Greenland coast is an example of a typical non-slip boundary to the ice flow along the coast (see Fig. 7).

EXAMINATION OF THE ANGLE OF INTERNAL FRICTION FOR SEA ICE

An ice field often exhibits a self-similar geometrical 

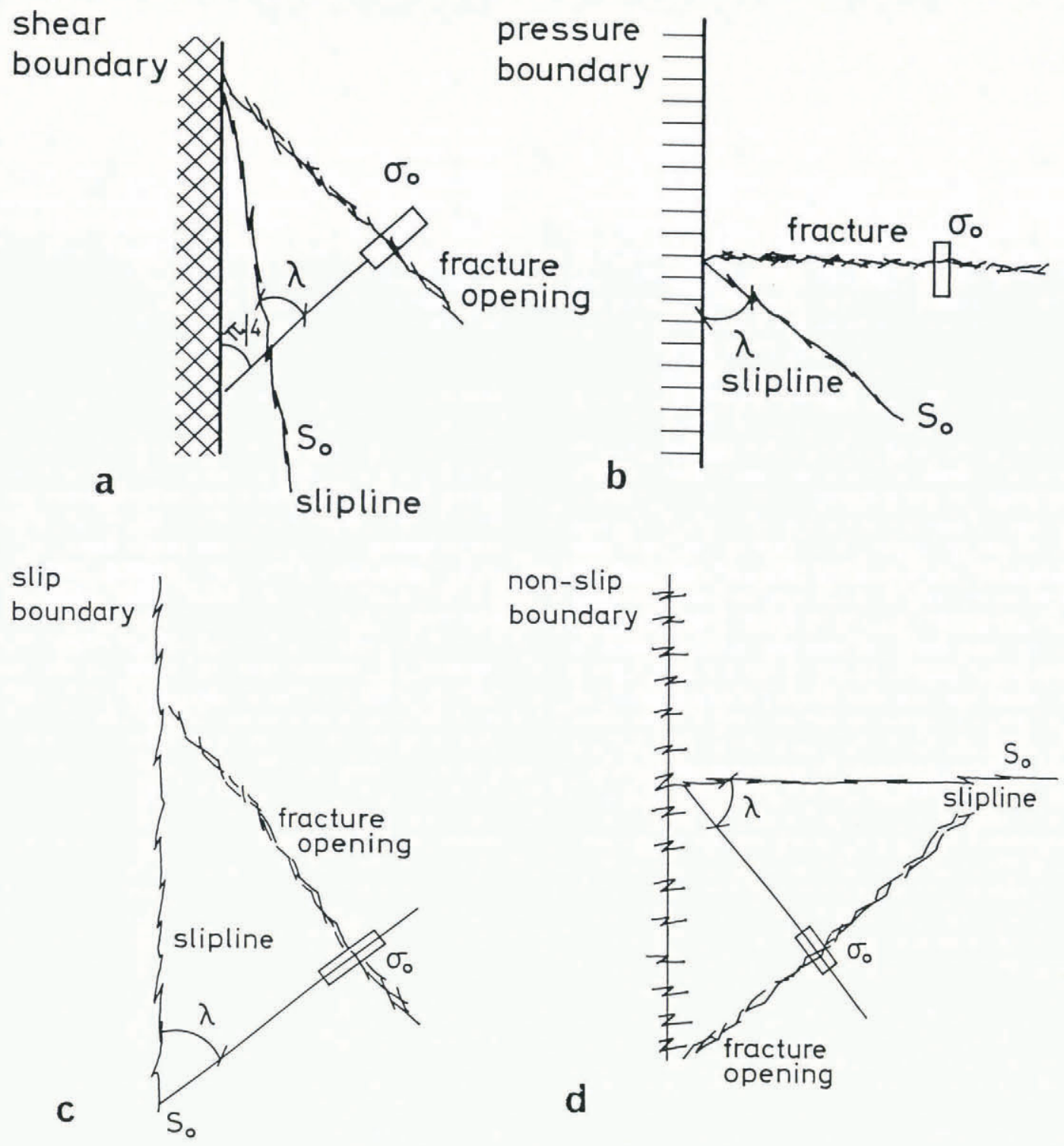

Fig. 6. Fractures and slip lines corresponding to the primary principal direction at different kinds of boundaries. Dynamic shear (a) and pressure (b) boundary. Kinematic slip (c) and non-slip (d) boundary.

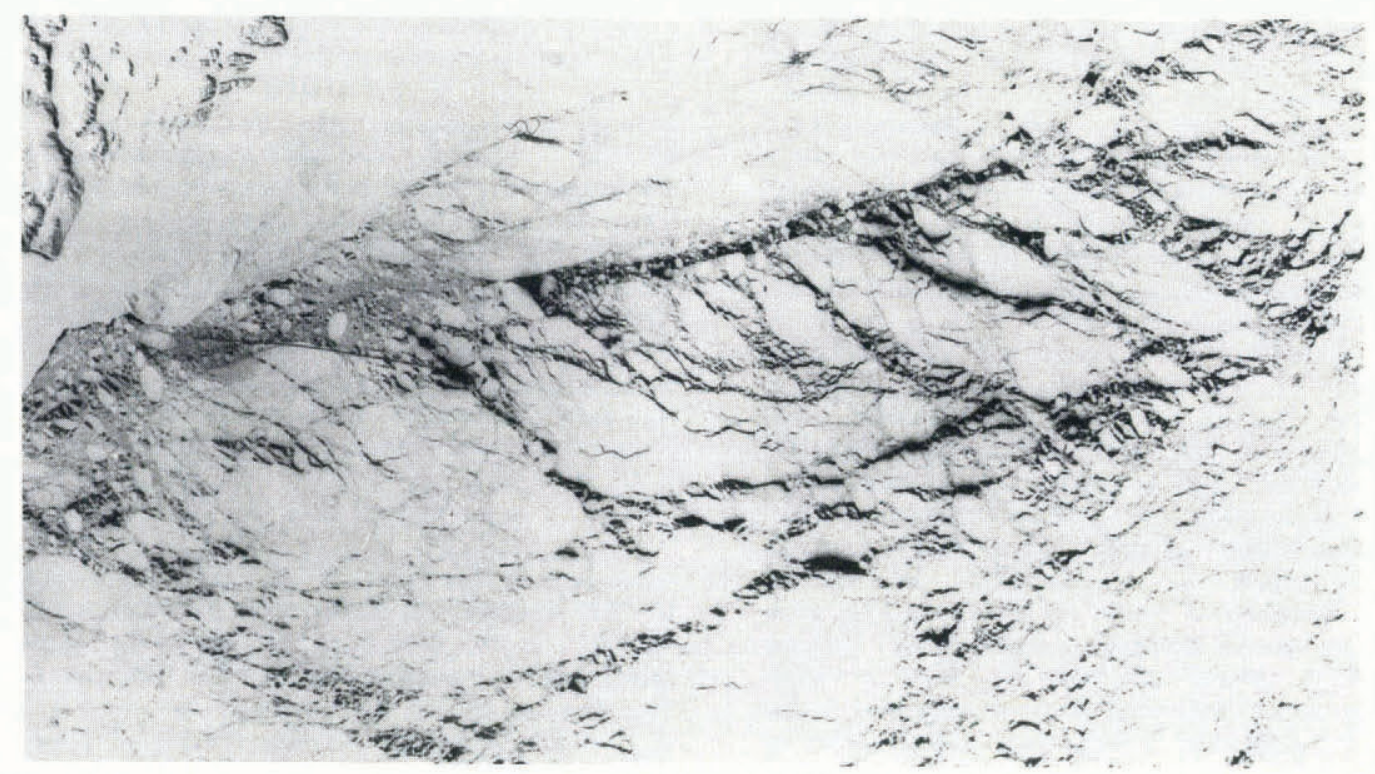

Fig. 7. Landsat image of the ice field off the Greenland coast, 25 April 1976. The length scale of the image is $185 \mathrm{~km}$ across. 
nature. The ice floes are fractured into a collection of objects which has scale-invariant, power-law dependent, cumulative-size distributions of geometrical quantities. Vertical sea-ice-keel profiles exhibit self-similar properties in a bounded regime of a particular horizontal length scale (Rothrock and Thorndike, 1980). The size distributions of various geometrical ice-floe quantities, such as ice-floe perimeter, diameter, and area (see for example Rothrock and Thorndike, 1984; Matsushita, 1985), and the slope of the spectral density of the ice-keel profiles can be described by the Hausdorff or fractal dimension $D$ characterizing this geometrical self-similarity. But the fractal dimension $D$ yields little quantitative information concerning the mechanical properties of the deformed material, unless something is said about the fractal generator of the fracture or deformation pattern. On the contrary, direct information about the mechanical properties is contained in the distribution of breaking angles. This distribution, the $\beta_{i}$ distribution, can be measured from distinguishable features on an arbitrary image of deformation.

Finding a mean value of the angle of internal friction from observed breaking angles is a process of trial and error. The deformation pattern being observed can represent different internal (stress-state) and external (loads or body forces) conditions, either past or present. Examining a unique value for the angle of internal friction from a particular deformation pattern might therefore turn out to be a difficult procedure. From a measured distribution of breaking angles, discrete values are represented by its peaks, which must be examined first. Eack peak gives rise to a set of values for $\Phi$ as a function of the breaking index $i$ (see Fig. 5). The number of different breaking indices is at least the same as the number of peaks. The examined value for the angle of internal friction is found from the permutation of indices, which leads to the same angle of internal friction corresponding to all of the respective sets. The following examination of the angle of internal friction is performed from measurements of breaking angles of observed deformation at different length scales in sea ice. This is given as a brief example for illustrating the application of the geometrical relationship derived above. In this case, the outer cut-off length scale, i.e. the observed maximum size of undeformed features, is denoted as the characteristic length scale of the observed deformation pattern.

The rectangular deformation patterns of newly frozen "finger-screwed" sea ice indicate plastic deformation behaviour and correspond to a nil value of the angle of internal friction $(\Phi=0)$. The observed patterns are characterized by distinct finger size ranging from 0.1 to $100 \mathrm{~m}$, which depends both on the ice thickness and the horizontal isotropic pressure. In contrast, the more common non-rectangular form of sea-ice features exemplifies the granular deformation behaviour and gives rise to a non-zero value for the angle of internal friction. Examples of these are large-scale features, which often range from 1 to $100 \mathrm{~km}$ and appear in systematic patterns that are commonly referred to as parallelograms or diamond-shaped patterns (e.g. Marco and Thomson, 1977; Vinje and Finnekảsa, 1986).

Measurements of the breaking angle of the most commonly observed parallelograms in the Beaufort Sea Gyre (Marco and Thomson, 1977) lead to an average breaking angle of $\beta=30^{\circ} \pm 10^{\circ}$, which belongs to a characteristic length scale of $100 \mathrm{~km}$. The shape factor (length divided by width) of the parallelograms frequently observed in Fram Strait (Vinje and Finnekåsa, 1986) correspond to a breaking angle of $B=30^{\circ} \pm 3^{\circ}$ which belongs to different characteristic scales ranging from 5 to $100 \mathrm{~km}$. An assemblage of values for the angle of internal friction, dependent on different breaking indices $i$, corresponds to this breaking angle $\left(\beta=30^{\circ}\right)$. The patterns corresponding to these different values (using Equation (10)) can be compared to the pattern obtained by measurements of sea-ice deformation. The comparison with observed deformation patterns, which were measured from Landsat images (Marco and Thomson, 1977), yields the best fit with a value $\Phi=15^{\circ} \pm 5^{\circ}$. The relative deformation was examined using the difference in the positions of recognized features after a $2 \mathrm{~d}$ time interval. The slip lines $\mathrm{S}_{0}, \mathrm{~S}_{1}$, and $\mathrm{S}_{2}$ are represented as lines which subdivide the sectors which are being rigidly displaced relative to a fixed point in the ice field. The orientation of nearest opening or lead corresponds to the primary principal direction, $\sigma_{0}$. Here the characteristic length scale is $100 \mathrm{~km}$.

The tracking of features on Seasat/SAR images (Fily and Rothrock, 1986) for the Central Arctic pack clearly demonstrates how slip lines occur in the sea-ice deformation field and where they systematically subdivide certain rigid elements. The elements were tracked between two different SAR scenes by means of a nested correlation algorithm which gave a resolution of $100 \mathrm{~m}$. The breaking angles (between slip lines) for two different images correspond to an angle of internal friction of $\Phi=14^{\circ} \pm 3^{\circ}$ (revolution 1382-1468) and $\Phi=15^{\circ} \pm 2^{\circ}$ (revolution 1439-1482). Here, the characteristic length scale is $100 \mathrm{~km}$.

Openings or leads of ten appear on satellite images as narrow zones of low ice concentration where the ice is severely broken (see for example the Landsat image of the ice field off the Greenland coast shown in Figure 7), because of the tensile stress and shearing displacements. The land-fast ice off the Greenland coast, where no deformation occurs, is considered as a non-slip boundary to the ice field farther off the coast. The angle of internal friction corresponding to the intersection angles of openings or leads, measured from the Landsat image (Fig. 7), is $\Phi=16^{\circ} \pm 1.5^{\circ}$. The characteristic length scale of this pattern is $10 \mathrm{~km}$.

A systematic pattern of shear ridges, belonging to a characteristic length scale of $1 \mathrm{~km}$, in the Baltic Sea ice field in April 1982 is shown in the areal photograph published in Lepparanta (1983, fig. 2). In this case, measurements of intersection angles between shear ridges lead to an angle of internal friction of $\Phi=15^{\circ} \pm 2^{\circ}$.

The ice-keel profiles must exhibit geometrical properties in accord with the processes that dictate their formation and deformation. The most important processes involved are freezing and melting, ridging and rafting, flexural breaking due to the presence of ocean swells or isostatic adjustment. Ridging and rafting are the results of compressive deformation in the horizontal plane that leads to shear strains in the vertical plane. When level ice of thickness $h$ undergoes compressive deformation, the departure of thickness from the level thickness $\Delta h$ is positive, and it follows the direction of maximum shear strain (i.e. the slip-line direction in the vertical plane). The shape of an ice-keel profile that is formed by compressive deformation is therefore in accord with the possible slip-line directions in the vertical plane.

A fundamental geometrical description of functions (discontinuous, fractal, or continuous) can be obtained from the exponent, $p$, of their spectral functions (Rothrock and Thorndike, 1980). Ice-keel profiles from the Central Arctic pack exhibit fractal properties in a horizontal wavelength regime (here called the fractal regime, i.e. $-3<p<-1$ ) between 20 and $200 \mathrm{~m}$. These limits indicate the inner and outer horizontal cut-off scales belonging to the vertical features that have resulted from granular deformation. A graphical examination (Fig. 6; Rothrock and Thorndike, 1980) of the spectral exponent of the ice-keel profile gives $p=-2.0 \pm 0.1$ in the fractal regime. The ice-keel profile exhibits continuous properties for wavelengths less than $20 \mathrm{~m}$ as the spectral exponent approaches $p \simeq-3$, and discontinuous properties for wavelengths greater than $200 \mathrm{~m}$ where the spectral exponent approaches $p \simeq-1$.

If one assumes that the relation between fractal dimension and the associated angle of internal friction (Equation (12)), corresponding to the simple generator of $2 \lambda$, is valid for vertical deformation of sea ice, then the relation between the spectral exponent $p$ and the fractal or Hausdorff dimension $D$ leads to a relation between the angle of internal friction and the spectral exponent, where:

$$
\sin (\lambda)=2^{-(p+3) /(p+5)} \text {. }
$$

Accordingly, the angle of internal friction that corresponds to the spectral exponent of the fractal regime of an ice-keel profile is $\Phi=15^{\circ} \pm 2.5^{\circ}$. The characteristic horizontal length scale of the vertical features here is $100 \mathrm{~m}$ This $\Phi$ value is the same (within the error of measurement) as that obtained from the distribution of breaking angles in the horizontal plane belonging to length scales $1-100 \mathrm{~km}$. The division of the ice-keel profile into regimes of widely different geometrical properties, continuous, fractal, and discontinuous, characterize the processes that dictate the 
formation of vertical features on various horizontal length scales. Continuous freezing and melting, fractal granular deformation, and discontinuous breaking due to swells or isostatic adjustment, could be a possible physical cause for these divisions.

\section{CONCLUSION AND DISCUSSION}

The purpose of this work has been to study the mechanisms of ice failure in order to explain the prominent systematic nature and self-similarity of deformation and fragmentation patterns. The results are concentrated in the simple geometrical relationship between the angle of internal friction and features in deformation patterns (Equation (11)). This geometrical relationship has been applied to find the angle of internal friction from a few selected deformation patterns in sea ice. The rectangular pattern of "fingerscrewed" new sea ice is an example of plastic deformation behaviour where the shear strength is constant (see Equation (6)). This plastic deformation behaviour usually concerns only a limited area of the sea-ice pack (refrozen leads) and its influence on the overall mechanical properties depends on the horizontal isotropic pressure and the thickness of this new ice. The analysis of the angle of internal friction leads one to the conclusion that the general mechanical behaviour of the sea-ice pack is granular on scales from $100 \mathrm{~m}$ to $100 \mathrm{~km}$ where $\Phi=15^{\circ} \pm 2^{\circ}$.

This result was not unexpected since the treatment of fragmented ice as a granular material conforms to the well-known Coulomb-Navier yield criterion, which has been done by many groups in an attempt to model the internal forces of the sea-ice pack. The values assumed or adopted from of ten ambiguous interpretations of experimental data (reviewed by Mellor, 1986) have been widely different with no preferred value. What is new in this work is that the angle of internal friction has been examined from direct observations of the deformation field and one has had the opportunity of studying how the mechanical properties are affected by different external and internal conditions. The results for sea-ice pack support the granular conceptual model for sea-ice pack (Thorndike, 1987), and the systematic correlations between features in the deformation field (Equation (10)) can be incorporated into it without altering the general concepts.

The method of finding the angle of internal friction from deformation data needs additional study and a more adequate algorithm will be developed. These results can be applied in a different manner. If the rheological properties are known a priori, then one can deduce from observations alone the conditions which give rise to a particular deformation pattern. This gives one the ability to distinguish between different deformation sequences, to measure the arcing (in the primary direction) between different places in the deformation field, and to examine the possibility of various features originating from identical external conditions.

Another example of the distribution of breaking angles is the direction distribution of lineaments in the crust of the planet Mercury. This deformation is due to tensile strains which arise from the shrinking and cooling of the crust (Thomas and Mason, 1983). The direction distribution of lineaments is found from the two-dimensional Fourier transform of the map of lineaments on the planet's surface. This distribution exhibits clear peaks of breaking angles that correspond to six different breaking indices and an angle of internal friction of $\Phi=25^{\circ} \pm 1^{\circ}$ (see Equation (13)). The fractal dimension of a simple generator of $2 \lambda$, corresponding to this angle of internal friction, is $D=1.32 \pm 0.02$. This fractal dimension is the same as the typical value $(D=1.3)$ found from morphological studies of the surface of the Earth (Mandelbrot, 1975). The fractal dimension found from the distribution of other geometrical quantities still has to be examined for comparison.

The evident self-similarity of fragmented and deformed sea ice on a wide range of scales (e.g. Rothrock and Thorndike, 1984; Matsushita, 1985) and the granular deformation behaviour exemplified above and shown here, i.e. $\Phi, D=$ constant, has its support in the framework of fractal geometry. A fractal generator of deformation patterns and its rule of placement must obey the same rule for geometrical properties as the features in the deformation field. One such generator is assumed to exist and with that a correspondence between the angle of internal friction and the fractal dimension, $D(\Phi)$ and $\Phi(D)$. The concentration (or density), $A(D, L)$, is considered as the only scale-invariant state variable, parameterized by the morphological parameters, fractal dimension, $D$, and the fragment size, $L$ (Mandelbrot, 1983). Thus, the definition of the angle of internal friction is expressed as

$$
\tan (\Phi(D))=-\left.\frac{\mathrm{d} f}{\mathrm{~d} \sigma_{\mathrm{n}}}\right|_{A(D(\Phi), L)} .
$$

This equation is only significant if $\Phi(D)=$ constant for all length scales $L$. In other words, granularity and self-similarity are scale-invariant properties of the deformation field and is interpreted equivalently within the framework of the fractal geometry.

The whole idea of ambient principal direction might seem confused from a continuum mechanics point of view. The ambiguity in principal direction introduced here reflects the alteration of the shear stress about the plane of weakness in materials when subjected to deformation. The concept of single principal direction, in a substance which is subject to deformation, applies only to the rigid elements in the deformation field. The principal directions of the rigid element, whose shapes and sizes are subject to one's own observational ability, accord with the possible principal directions (Equation (10)). One could ask: is it possible to define a single quantity related to the state of stress in a substance and consider it as the principal direction of the bulk (the overall collection of rigid elements)? The answer is: yes. This is the same kind of question addressed to any other continuum mechanics problem, where one wishes to relate the behaviour of the single elements to the behaviour of the bulk. In this case, one wants to find a single quantity which is common to all the principal directions of the rigid elements and to apply it as the principal direction of the bulk. The primary principal direction is common to all the rigid elements in a particular deformation field and it is ultimately related to the conditions at its external boundary. Accordingly, it is possible to achieve the appropriate choice or definition. It is both practical and physically significant to apply the primary principal direction to the principal direction of the bulk.

\section{SUMMARY}

The mechanism of failure and the geometrical properties of two-dimensional fragmentation and deformation in sea ice have been investigated. The Mohr formulation of failure, which is based on the concept of slip at the plane of weakness, is used in this study. The results concern the general geometrical relationship between features in deformation patterns and their relation to the properties of the material. Any feature which has a fixed angle to the principal direction $\sigma_{j}$ in a material, which is subject to deformation, has an orientation $\theta^{*}$ corresponding to the primary direction $\theta_{0}$ and the branching index $j$ where

$$
\theta_{j}^{*}=\theta_{0}+2 j \lambda ; \quad j=0, \pm 1, \pm 2, \ldots
$$

in fulfillment of the yield condition $\left|\tau_{\mathrm{n}}\right|=f\left(\sigma_{\mathrm{n}}, A_{i}\right)$. Here, $\tau_{\mathrm{n}}$ is the shear stress and $\sigma_{\mathrm{n}}$ is the normal stress at the plane of weakness and $A_{i}$ are the state variables. The corresponding angle of internal friction $\Phi$ and the angle $\lambda$ are defined as

$$
\tan (\Phi)=-\cot (2 \lambda) \equiv-\left.\frac{\mathrm{d} f}{\mathrm{~d} \sigma_{\mathrm{n}}}\right|_{A_{i}}
$$

This leads to the geometrical relationship between mechanically similar features (belonging to direction $\theta_{k}$ and $\theta_{l}$ ) which intersect with the angle

$$
\Delta_{i} \theta=\theta_{l}-\theta_{k}=2 i \lambda ; \quad i=(l-k)=0, \pm 1, \pm 2, \ldots
$$

and the corresponding breaking angle $\beta_{i}$ as a function of the angle of internal friction, and the breaking index $i$ is given by 


$$
B_{i}=\min \{|2 i \lambda-l \pi|\} ; \quad i, l=0, \pm 1, \pm 2, \ldots .
$$

The geometry represented by these equations gives rise to a simple fractal generator of $2 \lambda$ and corresponding Hausdorff dimension

$$
D=\ln (2) / \ln (2 \sin \lambda) .
$$

The examination of the angle of internal friction from horizontal deformation patterns in the Arctic Sea ice-pack (and one of shear ridges in the Baltic Sea) was done by using characteristic length scales of $1-100 \mathrm{~km}$ and resulted in the same $\Phi$ value within the error of the measurements, i.e. $\Phi=15^{\circ} \pm 2^{\circ}$. The deformation studied was in the systematic patterns of shear ridges, leads, and slip lines. These results support the theme of the granular conceptual model for the bulk properties of sea-ice pack (Thorndike, 1987). The wavelength regime of the vertical ice-keel profiles from the Central Arctic pack (Fig. 6; Rothrock and Thorndike, 1980), which are generated by compressive deformation in the horizontal plane, exhibits fractal properties between 20 and $200 \mathrm{~m}$. The angle of internal friction associated with the fractal dimension of $2 \lambda$ that has the same fractal dimension as the vertical features in sea ice is $\Phi=15^{\circ} \pm 2.5^{\circ}$.

The self-similarity of fragmented and deformed ice fields (e.g. Rothrock and Thorndike, 1984; Matsushita, 1985), and the strong evidence of granular deformation behaviour, can be mutually explained within the framework of the fractal notion. The fractal generator of the fracture or deformation pattern must obey the same geometrical rule as any feature in the deformation pattern. By considering the scale-invariant concentration (or density), $A(D, L)$, as the only state variable parameterized by the fractal dimension, $D$, and the fragment size, $L$, then the definition of the angle of internal friction is written as

$$
\tan (\Phi(D))=-\frac{\mathrm{d} f}{\mathrm{~d} \sigma_{\mathrm{n}}} \mid A(D(\Phi), L)
$$

This equation is only significant if $\Phi(D)=$ constant for all length scales $L$. In other words, granularity and self-similarity are scale-invariant properties of the deformation field and can be interpreted equivalently.

The ambiguity in principal direction introduced here reflects the alteration of the shear stress about the plane of weakness when straining or deformation occurs. The primary principal direction is common for all the rigid elements in a particular deformation field, and it is ultimately related to the conditions at its external boundary. It is both practical and physically significant to consider the primary principal direction as the principal direction of the bulk.

\section{ACKNOWLEDGEMENTS}

Most of this work was done while the author held a research scholarship from the Nordic Ministerial Council. I wish to thank $\mathrm{Mr} \mathrm{T}$. Vinje for support during this work and, by acceding to my claim that a mechanical explanation must exist, he initiated this study. I also wish to thank $\mathrm{Mr}$ D. Rothrock, who in the early stages of the study provided a deformation image (from SEASAT/SAR-imagery data) which encouraged my belief in what I was doing. Finally, I am particularly grateful to $\mathrm{Mr} \mathrm{J}$. Wettlaufer, who generously and carefully read the manuscript and contributed to many fruitful discussions and made many suggestions during the writing.

\section{REFERENCES}

Fily, M. and D.A. Rothrock. 1986. Extracting sea ice data from satellite SAR imagery. IEEE Trans. Geosci. Remote Sensing, GE-24(6), 849-854.

Jaeger, J.C. 1969. Elasticity, fracture and flow with engineering and geological applications. London, Chapman and Hall.

Kingston, M.R. and A.J.M. Spencer. 1970. General yield conditions in plane deformations of granular media. $J$. Mech. Phys. Solids, 18, 233-243.

Leppäranta, M. 1983. Size and shape of ice floes in the Baltic Sea in the spring. Geophysica, 19(2), 127-136.

Mandelbrot, B.B. 1975. Stochastic models for the Earth's relief, the shape and the fractal dimension of the coastlines and the number-area rule for islands. Proc. Natl. Acad. Sci. U.S.A., 72(10), 3825-3828.

Mandelbrot, B.B. 1983. The fractal geometry of nature. San Francisco, Freemann.

Marco, J.R. and R.E. Thomson. 1977. Rectilinear leads and internal motions in the ice pack of the western Arctic Ocean. J. Geophys. Res., 82(6), 979-987.

Matsushita, M. 1985. Fractal viewpoint of fracture and accretion. J. Phys. Soc. Japan, 54(3), 857-860.

Mellor, M. 1986. Mechanical behavior of sea ice. In Untersteiner, N., ed. The geophysics of sea ice. New York, Plenum Press, 165-281. (NATO ASI Ser., Ser. B Physics, 146.)

Nye, J.F. 1952. The mechanics of glacier flow. J. Glaciol., 2(11), 82-93.

Rothrock, D.A. and A.S. Thorndike. 1980. Geometric properties of the underside of sea ice. J. Geophys. Res., 85(C7), 3955-3963.

Rothrock, D.A. and A.S. Thorndike. 1984. Measuring the sea ice floe size distribution. J. Geophys. Res., 89(C4), 6477-6486.

Thomas, P.G. and P. Masson. 1983. Tectonic evolution of mercury; comparison with the Moon. Ann. Geophys., 1(1), 53-58.

Thorndike, A.S. 1987. A random discontinuous model of sea ice motion. J. Geophys. Res., 2(C6), 6515-6520.

Vinje, T. and Ø. Finnekasa. 1986. The ice transport through the Fram Strait. Nor. Polarinst. Skr. 186. 